\title{
New dynamic permeability upscaling method for flow simulation under depletion drive and no-crossflow conditions
}

\author{
Mohammad Sharif* and Mohan Kelkar \\ McDougall School of Petroleum Engineering, The University of Tulsa, 800 South Tucker Drive, Tulsa, Oklahoma, 74104, \\ United States \\ (C) China University of Petroleum (Beijing) and Springer-Verlag Berlin Heidelberg 2013
}

\begin{abstract}
The main purpose of upscaling in reservoir simulation is to capture the dynamic behavior of fine scale models at the coarse scale. Traditional static or dynamic methods use assumptions about the boundary conditions to determine the upscaled properties. In this paper, we show that the upscaled properties are strongly dependent on the flow process observed at the fine scale. We use a simple nocrossflow depletion drive process and demonstrate that an upscaled property is not a constant value. Instead, if the goal is to match the performance of the fine scale model, the upscaled permeability changes with time. We provide an analytical solution to determine the upscaled permeability and present the value of upscaled permeability under limiting conditions. Our equation suggests that it is possible that upscaled value can fall outside the range of fine scale values under certain conditions. We show that for pseudo steady state flow, using common averaging methods like arithmetic or even geometric averaging methods can lead to optimistic results. We also show that the no-crossflow solution is significantly different than crossflow solution at late times. We validate our method by comparing the results of the method with flow simulation results in two and multi-layered models.
\end{abstract}

Key words: Upscaling, permeability, reservoir simulation, no-crossflow, depletion drive

\section{Introduction}

Reservoir simulation allows an engineer to evaluate various complex scenarios and predict the future reservoir performance. With increasing computer power and better geological and geophysical evaluation, it is possible to obtain more detailed information about reservoirs. Instead of building overly simplistic models only capturing extremely large scale details, geo-modelers can build a very detailed model capturing small scale details which can resolve what can be observed on a core scale. These detailed models contain millions of discrete cells and running flow simulations on these fine models for multiple scenarios is practically impossible. More than that, production data resolution is much coarser than the static data. Because of these two limitations, the static model (or geo-cellular model) needs to be upscaled to a dynamic (coarse simulation) model so that it can serve two purposes: (i) it can reduce the computational effort to conduct simulation; and (ii) it can capture the resolution of dynamic data.

This illustrates the difficulty in upscaling the geo-cellular models. First, upscaling by necessity, would be dependent on

*Corresponding author. email: mohammad-sharifi@utulsa.edu Received May 20, 2012 the flow process we are trying to mimic. i.e. for petroleum reservoirs, a simple depletion process will require different upscaling than gravity dominated displacement. Second, it would be computationally very expensive to simulate the geo-cellular model under actual flow process conditions to compare it to the dynamic (coarse scale) model.

In this paper, we consider a simple depletion process in a petroleum reservoir to illustrate the difficulty in upscaling reservoir properties using simplified approaches. By using an analytical approach, we propose a method for calculating upscaled permeability for depletion processes.

\section{Literature review}

While reducing the number of grid blocks in a geo-cellular model to create a dynamic model, we normally go through a two step process: upgridding and upscaling. In the first step, we determine to what extent we can coarsen the geocellular model (Kelkar and Sharifi, 2012). For example, if we had five hundred fine scale layers in the geo-cellular model, we determine that reducing those layers to about fifty would retain enough geological details. Then we should determine how to upscale the reservoir properties in the upscaled model. For example, if we combine a certain number of layers into one upscaled layer, we will need to define an effective 
property of that single layer by "averaging" the properties of corresponding fine scale layers. In petroleum reservoir, the two most common properties which are upscaled are: porosity and permeability. Porosity is usually upscaled by volume weighted arithmetic averaging for preserving the original hydrocarbon in place but there is not a unique solution for permeability upscaling (Eberhard et al, 2002; Wu et al, 2004; Salazar and Villa, 2007; Chen 2009). In this paper, we are only concentrating on upscaling of reservoir permeability. We are assuming that upgridding step has already been conducted.

Among different petrophysical properties we upscale, estimating single equivalent permeability for a set of fine scale grids with different permeabilities is a challenging problem (Wen and Gómez-Hernández, 1996; Li et al, 2001; Locka et al, 2004; Durlofsky, 2005; Babaei and King, 2011). Many simple analytical approaches (i.e. harmonic, geometric and arithmetic) have been suggested by researchers depending on flow direction and heterogeneity of reservoir (Muskat, 1937; Cardwell and Parsons, 1945; Craft and Hawkins, 1959; Warren and Price, 1961).

Russell and Prats (1962) investigated the impact of cross flow on upscaled properties. They demonstrated that - in the presence of cross flow - an arithmetic upscaling of permeability is appropriate and is able to represent the behavior of fine scale layers. They also demonstrated that the early time behavior under cross flow and no-crossflow conditions is similar; however, at late times, the behavior is significantly different. In this paper, our emphasis is on layers producing under no cross flow conditions.

Besides the usual statistical averaging methods (harmonicgeometric-arithmetic) the renormalization method was developed in the 1980s. It was based on the concept of a resistor network (Wilson, 1979; Shah and Ottino, 1986; King, 1989; Mohanty and Sharma, 1990; Kelkar et al, 2002). Unfortunately, this method did not work well for strongly anisotropic porous media. Malick (1995) showed that block permeabilities obtained through renormalization could have even a $200 \%$ error in the case of heterogeneous sand shale combinations.

Deutsch (1985) and Journel et al (1986) proposed the use of a power averaging method to compute equivalent permeability as Eq. (1):

$$
k_{\text {eff }}=\left(\frac{1}{v} \int_{v} k_{i}^{p}(u) \mathrm{d} u\right)^{1 / p}
$$

where $k_{\text {eff }}$ is the effective permeability, $\mathrm{mD} ; k_{i}$ is the permeability of layer $i, \mathrm{mD} ; u$ is the integral variable, $\mathrm{ft}^{3} ; v$ is the volume of interest, $\mathrm{ft}^{3} ; p$ is the power averaging exponent.

Eq. (1) is general and by changing the exponent $p$ between $-1,0$ and 1 , the power average changes between harmonic, geometric and the arithmetic averages respectively. Although the power averaging method can be customized for a specific scenario, determining the value of $p$ proved to be difficult in practical cases. Without conducting simulations of both fine scale and coarse scale models and comparing the results, it is difficult to determine the value of $p$.

Unlike static methods discussed above, dynamic methods use dynamic processes to capture the upscaled values (Durlofsky, 2005). In the dynamic method the effective permeability is assigned in a way that it can reproduce the same flux that was passing through the fine scale models. However, the result of effective permeability depends on the boundary conditions that should be imposed on the volume of interest. No-flow, constant pressure, linear pressure and periodic pressure boundary conditions can be applied to the volume of interest and different results can be obtained. Due to the necessity of solving diffusivity equations with different boundary conditions, dynamic methods are computationally demanding compared to static methods. The values obtained can change with boundary conditions. Choosing appropriate volumes could also be a problem in the case of the presence of isolated sand and shale bodies (Durlofsky, 2005; Stone et al, 2007). It is also known that instead of upscaling permeabilities, sometime it is better to upscale transmissibilities to properly capture the flow behavior of a fine scale model.

By examining the literature, one common theme in all the methods is that these methods are not based on the type of flow process we are trying to capture in the upscaled model. All these methods are based on single phase flow and most of them assume steady state flow conditions.

\section{Problem statement}

To summarize the current state of the art in upscaling, the static based methods are computationally efficient but may not reproduce dynamic characteristics of the fine scale model well; whereas, the dynamic methods are computationally demanding and may not provide optimal upscaling without precise knowledge of the well configuration as well as the assigning of appropriate boundary conditions. Our goal in this research is to demonstrate the importance of flow processes on upscaling permeability of the coarse model. We consider a simple flow process of depletion in a layered reservoir. We further assume a no-crossflow condition. Our goal in this research is to concentrate on dynamic behavior of the fine scale model under depletion conditions. We want to determine the effective property of the coarse scale model so that the depletion process - observed under fine scale conditions - is honored in the coarse scale model.

\section{Proposed method/hypothesis}

The main hypothesis in the current methodology is that effective permeability of a coarse scale model should be defined in a way that it can give us similar cumulative production as observed in the fine scale model. We only consider depletion processes under no-crossflow conditions in a layered reservoir. We assume pseudo-steady state flow during the depletion process and calculate the effective permeability using an analytical process. We then compare the solution we obtained with the flow simulation results. We further extend our analysis to a fourteen-layered model. 


\subsection{Approach}

We consider a simple, two-layered model, which is filled with oil. This is shown in Fig. 1.



Fig. 1 Schematic of the simple two-layer model

The two layers have different permeability and porosity values. A single well is penetrating both the layers and is producing at a constant bottom hole pressure. Porosity is upscaled using a volume-weighted arithmetic average. Our goal is to find an effective permeability of the upscaled model (combination of two layers) such that cumulative production from two layers is similar to cumulative production from an upscaled model. Mathematically, we can write:

$$
\int_{0}^{t}\left(q_{1}(t)+q_{2}(t)-q_{\text {eff }}(t)\right) \mathrm{d} t=0
$$

where $q_{1}(\mathrm{t})$ represents the rate at which oil is being produced from the first layer, $q_{2}(\mathrm{t})$ represents the rate at which oil is being produced from the second layer, and $q_{\mathrm{eff}}(\mathrm{t})$ is the rate at which oil is being produced from the upscaled reservoir, $\mathrm{STB} / \mathrm{d} ; t$ is time, day.

We assume pseudo-steady flow. Based on our assumptions, we can determine the effective permeability of the upscaled model depending on the cumulative time. The detailed derivation is provided in the Appendix. At the late time, the effective permeability of two layers combination can be written as:

$$
k_{\text {eff }}=\left(\frac{k h}{v_{\mathrm{p}}}\right)_{\min } \frac{v_{\mathrm{p} 1}+v_{\mathrm{p} 2}}{h_{1}+h_{2}}
$$

where min index represents a layer with a smaller value of $k h / v_{\mathrm{p}}$, this value is equivalent to $k / \varphi$ for most of the cases; $k$ represents the permeability, $\mathrm{mD} ; h$ represents the thickness, $\mathrm{ft} ; \varphi$ represents the porosity; $v_{\mathrm{p} 1}$ and $v_{\mathrm{p} 2}$ are hydrocarbon pore volumes of layers 1 and 2 , respectively; $\mathrm{ft}^{3}$. At early times, we prove that effective permeability can be written as:

$$
k_{\text {eff }}=\frac{k_{1} h_{1}+k_{2} h_{2}}{h_{1}+h_{2}}
$$

That is, at early times, the effective permeability is the arithmetic average; however, at late times, porosity (pore volume) also plays an important role in determining the effective permeability. Among the two equations, Eq. (3) provides some interesting insights. For example, if $k / \varphi$ for both the layers is the same, Eq. (3) reduces to Eq. (4). I.e. assume that $k_{1} / v_{\mathrm{p} 1}=k_{2} / v_{\mathrm{p} 2}$ and choose $\min =1$. Therefore we can obtain

$$
\begin{aligned}
k_{\mathrm{eff}} & =\left(\frac{k h}{v_{\mathrm{p}}}\right)_{1} \frac{v_{\mathrm{p} 1}+v_{\mathrm{p} 2}}{h_{1}+h_{2}}=\frac{k_{1} h_{1} v_{\mathrm{p} 1}+k_{1} h_{1} v_{\mathrm{p} 2}}{\left(h_{1}+h_{2}\right) v_{\mathrm{p} 1}} \\
& =\frac{k_{1} h_{1} v_{\mathrm{p} 1}+k_{2} h_{2} v_{\mathrm{p} 1}}{\left(h_{1}+h_{2}\right) v_{\mathrm{p} 1}}=\frac{k_{1} h_{1}+k_{2} h_{2}}{h_{1}+h_{2}}
\end{aligned}
$$

This means that the effective permeability is constant throughout the depletion process and is equal to arithmetic average if $k / \varphi$ for both layers is the same. On the other extreme, it is possible that the effective permeability at late times could be even smaller than the smallest fine model permeability value. Assume that the first layer has the higher permeability $\left(k_{1}>k_{2}\right)$ and consider a limiting case where the pore volume for the high permeability layer is significantly smaller than pore volume of the low permeability layer $\left(v_{\mathrm{p} 1}<<v_{\mathrm{p} 2}\right)$. Then according to Eq. (3) the effective permeability of the upscaled layer at the late times will be equal to:

$$
k_{\text {eff }}=\left(\frac{k h}{v_{\mathrm{p}}}\right)_{2} \frac{0+v_{\mathrm{p} 2}}{h_{1}+h_{2}}=\frac{k_{2} h_{2}}{h_{1}+h_{2}}
$$

For a depletion process, if the high permeability layer does not contribute much in terms of production at a later stage but adds thickness only, to match the performance of the reservoir, the effective permeability has to be smaller than the permeability of the lowest permeability layer. This result is significantly different than other methods since all the upscaling methods assume that the effective property is always going to fall within the limits of fine scale values. What Eq. (3) demonstrates is that it is possible that the effective property could be outside the range of fine scale values.

One important observation is that the upscaled permeability under no-crossflow conditions is significantly different than under cross flow conditions. Our analytical solutions (no-crossflow) differ significantly from Russel and Prats (in the presence of crossflow) solution at late times. The reason that we investigated the no-crossflow assumption is because in tight sand reservoirs with significant presence of shale layers (more than $80 \%$ ), no-crossflow is a much more realistic assumption in predicting the performance of the reservoir. It was observed that using arithmetic averaging for permeability upscaling in single phase flow, even in the case of the optimum layering scheme, will cause overestimation of the production profile (Sharifi and Kelkar, 2012).

For testing the analytical results, we consider a twolayer model with the permeabilities of 100 and $10 \mathrm{mD}$, respectively. The porosity of the first layer is 0.1 . The porosity of the second layer is varied as shown in Table 1. First and second columns of Table 1 show different porosity values for the second layer and late time limit for effective permeability respectively.

Table 1 A comparison for different upscaling methods for different cases

\begin{tabular}{cccccc}
\hline Case & $\begin{array}{c}\text { Late time } \\
\text { effective } \\
\text { permeability } \\
\mathrm{mD}\end{array}$ & $\begin{array}{c}\text { Transmissibility } \\
\text { multiplier }\end{array}$ & $\begin{array}{c}\text { Arithmetic Geometric } \\
\text { error } \\
\%\end{array}$ & $\begin{array}{c}\text { Arithmetic }+ \\
\text { error } \\
\text { transmissibility } \\
\text { multiplier error } \\
\%\end{array}$ & $\begin{array}{c}\text { mor } \\
\varphi_{2}=0\end{array}$ \\
50 & 0.91 & 0.7 & 8 & 0.6 \\
$\varphi_{2}=0.01$ & 55 & 1 & 0.1 & 5 & 0.1 \\
$\varphi_{2}=0.1$ & 10 & 0.2 & 20 & 11 & 7 \\
$\varphi_{2}=0.5$ & 6 & 0.11 & 123 & 79 & 23 \\
\hline
\end{tabular}


For all the four cases listed in Table 1, in Fig. 2 we show the flow simulation comparison between fine and upscaled models using arithmetic averaging, geometric averaging and late time permeability approximation. We first run a fine scale, two-layer model, using different permeability and porosity values for each layer (Fig. 3). We then combine the two layers into one by ensuring that the pore volume of the combined layer matches with the total pore volume of two layers. We use different averaging methods for upscaled permeability and run the simulation. Dynamic error is defined as an absolute value of difference between production as a function of time from fine and coarse scale models. It is clear that for the cases where the effective permeability result from Eq. (3) is smaller than the arithmetic average $(55 \mathrm{mD})$, the dynamic error also is greater (fourth column of Table 1). For better comparison, in addition to arithmetic averaging, dynamic error from geometric permeability upscaling is also given in the fifth
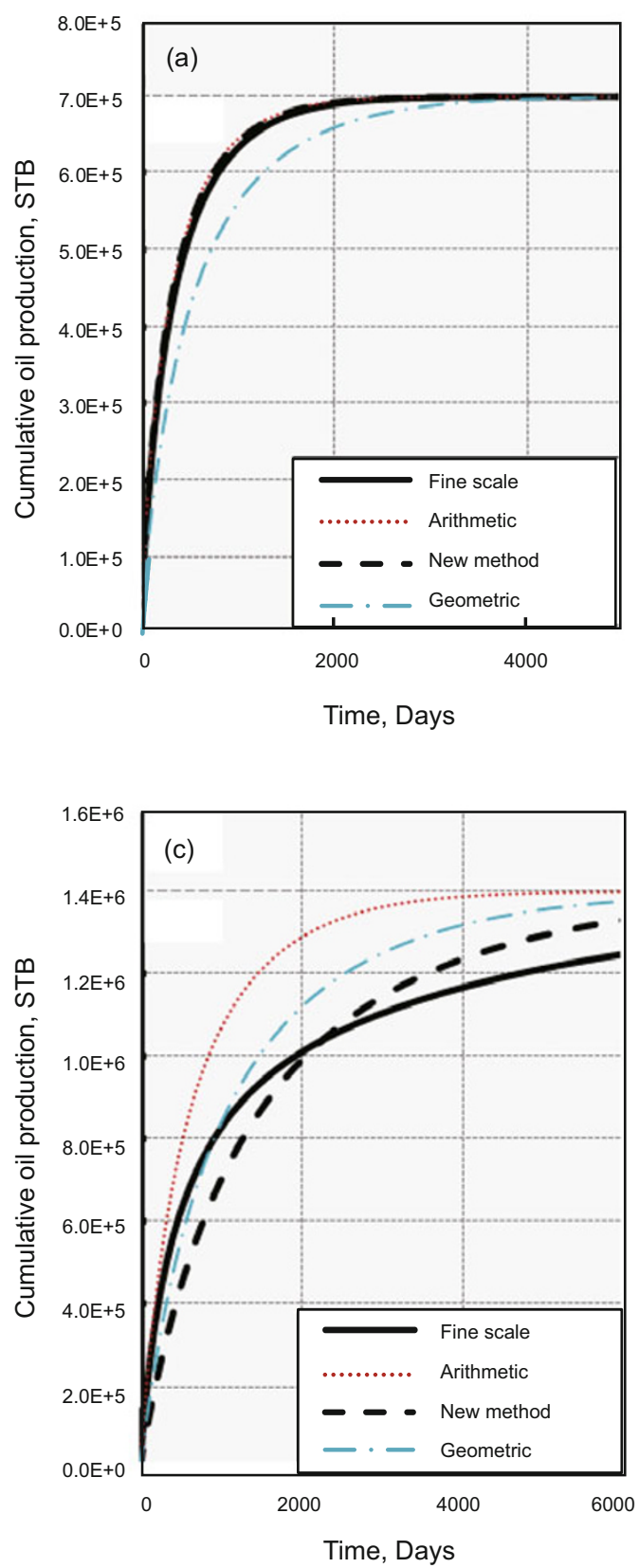

column of Table 1. The new method (which uses the late time value) provides much closer result when comparing fine scale production with coarse scale production. In our method, the effective permeability changes with time. However, we observed that for most cases, for bulk of the relevant time, the effective permeability is closer to the limiting value at late times. Rather than using a varying permeability value, which would be time dependent and difficult to implement, we used a limiting value for comparing our results with other methods. It should be mentioned that in the new method, we did not change the permeability of the formation. Instead, we used transmissibility multiplier in a coarse scale model to account for new permeability. This is to ensure that the productivity of the well is not affected by new permeability modification. In effect, we calculated the upscaled permeability using an arithmetic average. We then calculated the permeability using the new method and determined what transmissibility value
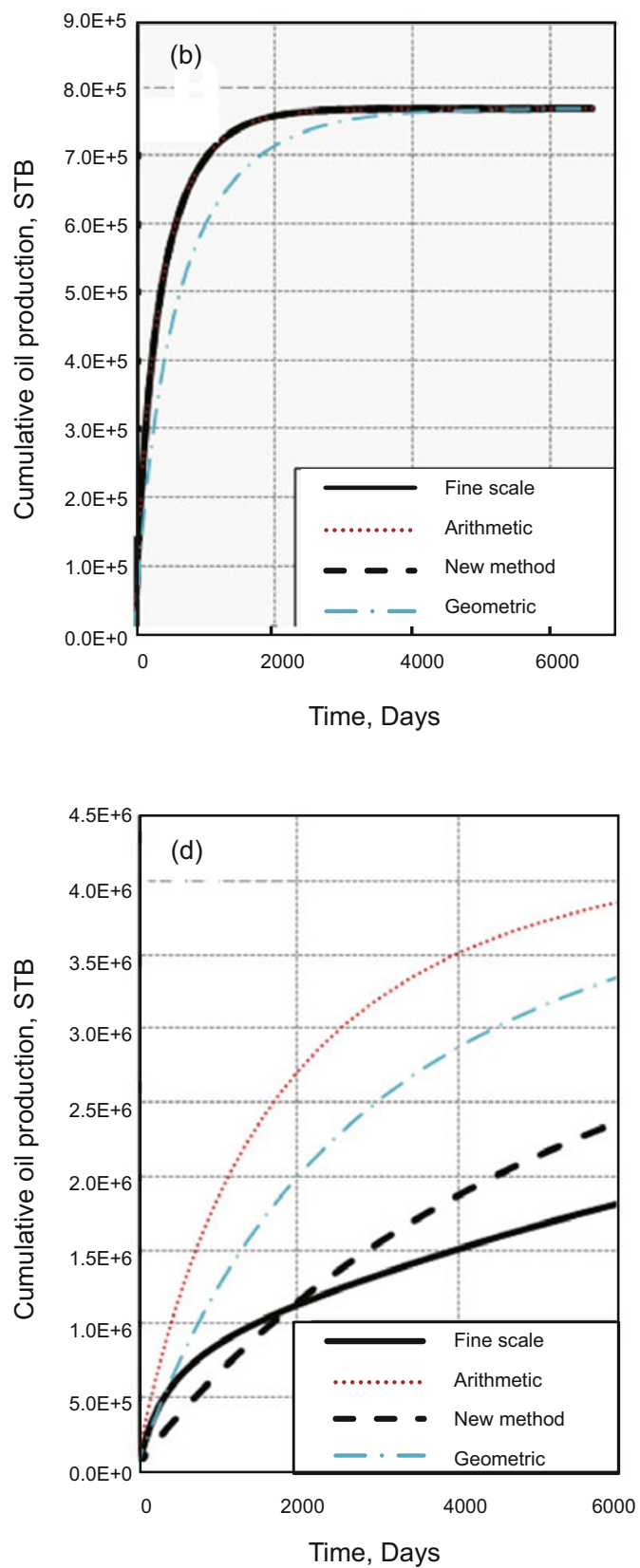

Fig. 2 Flow simulation comparison between fine and coarse scale models for different porosities (a) $\varphi_{2}=0$, (b) $\varphi_{2}=0.01$, (c) $\varphi_{2}=0.1$, and (d) $\varphi_{2}=0.5$ 


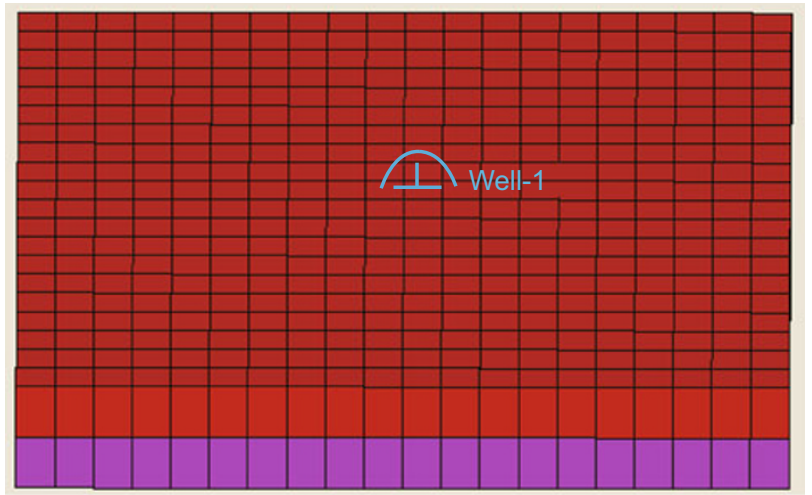

Fig. 3 Two-layer model for running fine scale numerical simulation

we would have obtained if we had used the new permeability value. Then we determined the multiplier to adjust the transmissibility of the arithmetically averaged model.

Ideally, based on analytical results, we should change the permeability as a function of time which is not a practical solution. Fig. 4 shows the analytically derived normalized cumulative production (green line) and effective permeability (red dash line) for different cases as a function of time on a semi-log scale. Cumulative production and effective permeability were calculated from Eq. (8A) and Eq. (13A) in the appendix respectively. It can be seen that for all the cases the effective permeability varies between the value of Eq. (4) and Eq. (3). In most cases, we observed that, by using a late time value (Eq. (3)), we could match the production results reasonably well. This is because for bulk of the time, the upscaled value is closer to the limiting, late time value. Please note that the $x$ axis is logarithmic in Fig. 4.

\subsection{4-layer model}

In this section, a 14-layer model is used to test the prediction using our method. The permeability values of these layers from top to bottom are 200, 10, 25, 1, 1,200, 1,000, 25, $20,30,40,30,45,1$ and $2 \mathrm{mD}\left(k_{1}\right.$ to $\left.k_{14}\right)$. The layer thickness and porosity values are the same. The pressure-based upgridding method can be used to find optimum gridding (Hosseini and Kelkar 2010). We combined 14 layers into five layers by combining $3,1,2,6$, and 2 layers respectively. In Fig. 5 the production results of the fine scale model and the result of the upscaled 5-layer model using different averaging methods are shown. Both arithmetic and geometric averaging methods overestimate the production during the entire production period. For testing our method we calculated effective permeability for each layer and then we used a transmissibility multiplier as discussed for the two-layer model case. First, effective permeability for each coarse layer was calculated sequentially. For example when we combined three layers together, first we calculated the effective permeability for first two layers $\left(k_{\mathrm{effl}, 2}\right)$ and then we have two layers, with a permeability of $k_{\mathrm{effl}, 2}$ and $k_{3}$ respectively.
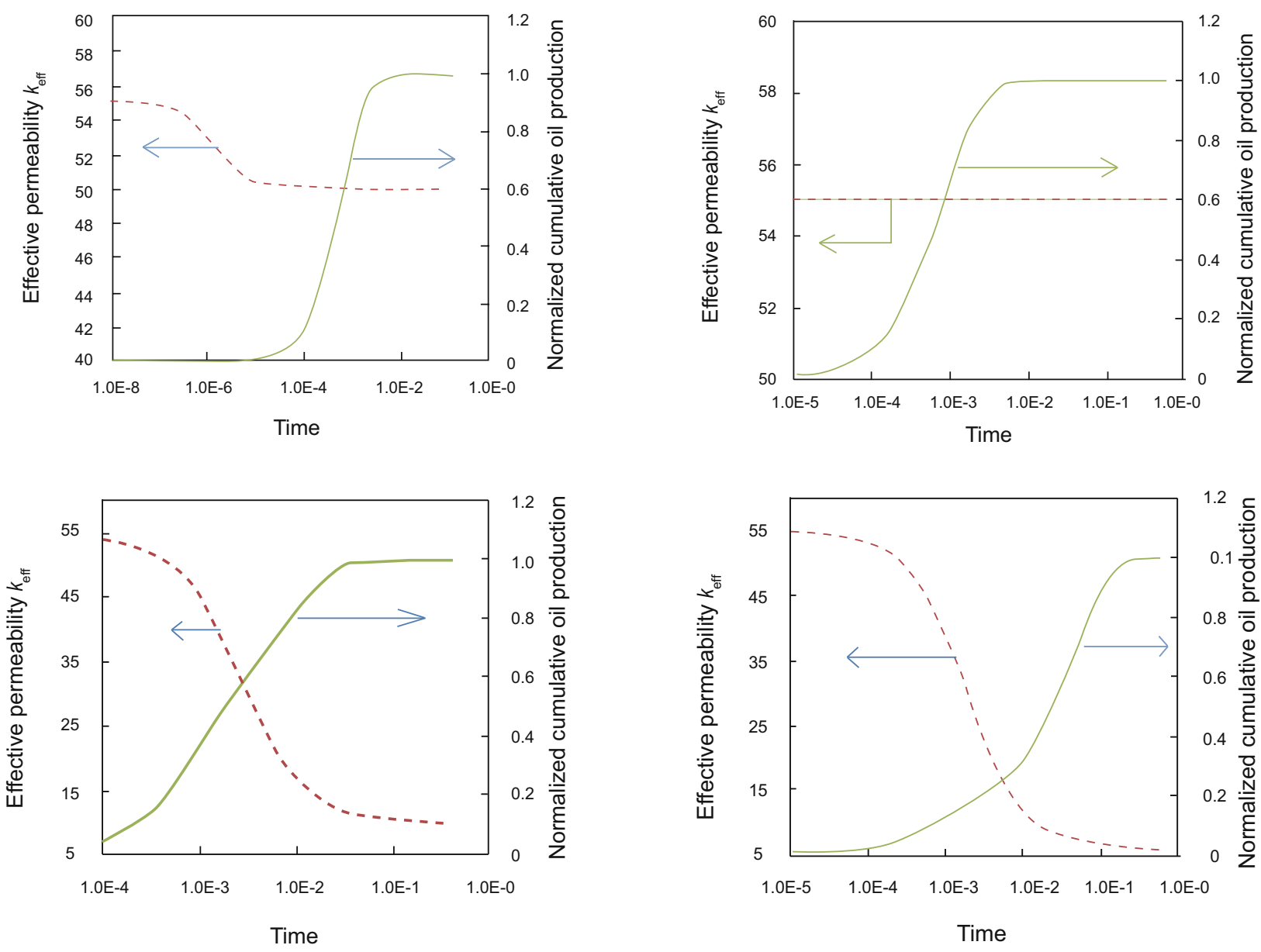

Fig. 4 Analytical calculated cumulative production and effective permeability for different porosity cases (a) $\varphi_{2}=0,(\mathrm{~b}) \varphi_{2}=0.01,(\mathrm{c}) \varphi_{2}=0.1$, and (d) $\varphi_{2}=0.5$ 


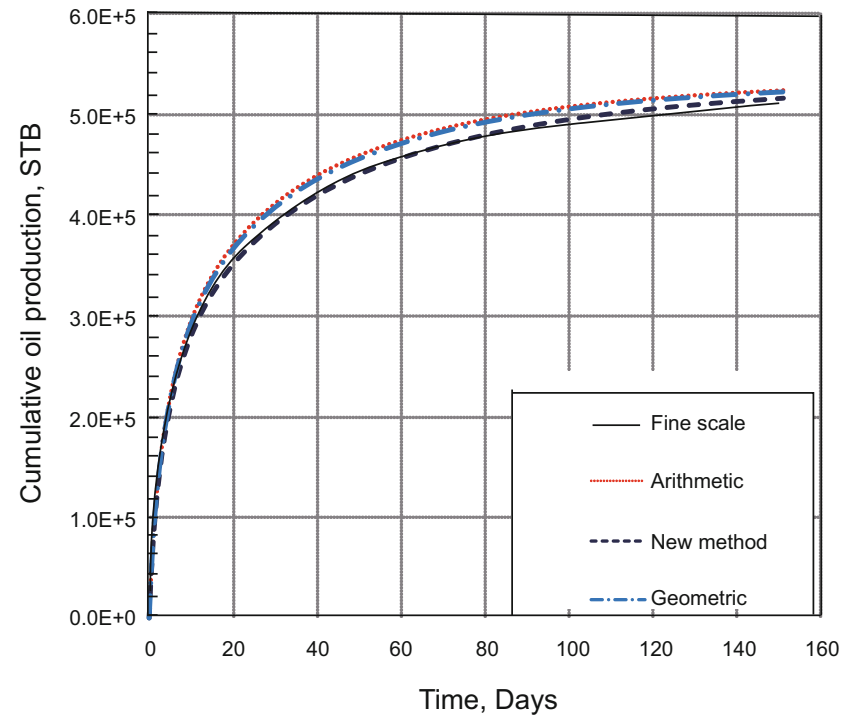

Fig. 5 A comparison of production profile for different averaging methods of the 14-layer model

In this step we can calculate the effective permeability for all three layers $\left(k_{\mathrm{effl}, 2,3}\right)$ using Eq. (3). After calculating effective permeability for all layers, then final transmissibility multiplier was calculated as ratio of calculated late time effective permeability to arithmetic average permeability. The reason why we did not use the effective permeability directly is that we know that very early time behavior of upscaled permeability is closer to arithmetic average (Eq. (A25)) and also we did not want to change the kh value for the wellbore. It is clear that by applying our methodology, the dynamic behavior of the fine scale model (here cumulative production) is captured better.

\section{Conclusions}

In this research, we demonstrated that upscaling of the effective permeability is dependent on time for a simple depletion process. By using a simple two-layer model, we developed an analytical expression for the effective permeability as a function of time. We showed that for later times, the effective permeability is not only a function of permeability of the fine scale model but also porosity of the fine scale model. It is possible that the effective permeability of the combined layers could be less than the individual permeabilities at later times. It was observed that commonly used arithmetic average upscaling method can provide us the optimal answer when the ratio of permeability to porosity of the fine scale layers is identical. In all other cases, arithmetic average overestimates the production. We validated our analytical method by comparing simulation results of a twolayer model with the analytical solution. We further extended our analysis to a multi-layered reservoir producing under depletion conditions. The results of this study demonstrated the importance of incorporating flow processes in defining upscaled properties. These results could be very useful in upscaling of tight reservoirs with significant presence of shale where no-crossflow is a much more realistic assumption in predicting the performance of the reservoir.

\section{References}

Babaei M and King P R. A comparison between wavelet renormalization upscaling methods and iterative upscaling-downscaling scheme. Paper SPE 141418 presented at the Reservoir Simulation Symposium, 21-23 February 2011, The Woodlands, Texas, USA

Cardwell W T and Parsons R L. Averaging permeability of heterogeneous oil sands. Journal of Petroleum Transaction, AIME. 1945. 160: 34-42

Chen T. New methods for accurate upscaling with full-tensor effects. Ph.D. Dissertation. Stanford University. 2009

Craft B C and Hawkins M F. Applied Petroleum Reservoir Engineering. Prentice Hall, New Jersey. 1959

Dake L P. Fundamental of Reservoir Engineering. Amsterdam: Elsevier. 1979

Deutsch C. Estimating block effective permeability with geostatistics and power averaging. Paper SPE 15991 presented at 1985

Durlofsky L J. Upscaling and gridding of fine scale geological models for flow simulation. The 8th International Forum on Reservoir Simulation, 20-24 June 2005, Stresa, Italy

Eberhard J, Attinger S and Wittum G. Coarse graining for upscaling of flow in heterogeneous porous media. Multiscale Modeling and Simulation. 2004. 2(2): 269-301

Fetkovich M J. Decline curve analysis using type curves. Journal of Petroleum Technology. 1980. 32(6): 1065-1077

Hosseini S A and Kelkar M. Analytical upgridding method to preserve dynamic flow behavior. SPE Reservoir Evaluation \& Engineering. 2010. 3(3): 473-484

Journel A G, Deutsch C and Desbarats A J. Power averaging for block effective permeability. Paper SPE 15128 presented at SPE California Regional Meeting, 2-4 April 1986, Oakland, California

Kelkar M, Perez G and Chopra A. Applied Geostatistics for Reservoir Characterization. SPE, Richardson, TX, 2002

Kelkar M and Sharifi M. Upgridding under multiphase flow conditions. Paper SPE 151043 presented at Improved Oil Recovery Symposium, 14-18 April 2012, Tulsa, Oklahoma, USA

King P R. The use of renormalization for calculating effective permeability. Transport in Porous Media. 1989. 4(1): 37-58

Li D, Beckner B and A Kumar. A new efficient averaging technique for scaleup of multimillion-cell geologic models. SPE Reservoir Evaluation \& Engineering. 2001. 4(4): 297-307

Locka P A, Jing X and Zimmermana R W. Comparison of methods for upscaling permeability from the pore scale to the core scale. Journal of Hydraulic Research. 2004. 42(Supplement 1): 3-8

Malick K M. Boundary effects in the successive upscaling of absolute permeability. Master Thesis. Stanford University, CA, 1995

Mohanty S and Sharma M M. A recursive method for estimating single and multiphase permeabilities. Paper SPE 20477 presented at the 65th Annual Technical Conference and Exhibition, 23-26 September 1990, New Orleans, Louisiana, USA

Muskat M. The Flow of Homogenous Fluids Through Porous Media. McGraw-Hill, New York, 1937

Russel D G and Prats M. Performance of layered reservoirs with crossflow- single compressible-fluid case. SPE Journal. 1962. 2(1): 53-67

Salazar M O and Villa J R. Permeability upscaling techniques for reservoir simulation. Paper SPE 106679 presented at Buenos Aires, 15-18 April 2007, Buenos Aires, Argentina

Shah N and Ottino J M. Effective transport properties of disordered, multiphase composites: Application of real-space renormalization group theory. Chemical Enginering Science. 1986. 41(2): 283-296

Sharifi M and Kelkar M. New upgridding method to capture the dynamic performance of the fine scale heterogeneous reservoir. Journal of Petroleum Science and Engineering. 2012. 86-87: 225-236 
Stone M T, Wu X H, Parashkevov R R, et al. Challenges and solutions in global-flow-based scaleup of permeability: Isolated flow bodies. Paper SPE presented at the SPE Reservoir Simulation Symposium, 26-28 February, 2007, Houston, Texas, USA

Warren J E and Price H H. Flow in heterogeneous porous media. SPE Journal. 1961. 1(3): 153-169

Wen X H and Gómez-Hernández J J. Upscaling hydraulic conductivities in heterogeneous media: An overview. Journal of Hydrology. 1996. 183(1-2): ix-xxxii

Wilson K G. Problems in physics with many scales length. Scientific American. 1979. 241: 158-179

Wu X H, Efendiev Y and Hou T Y. Analysis of upscaling absolute permeability. Journal of Discrete and Continuous Dynamical Systems, Series B. 2002. 2(2): 185-204

\section{Appendix}

Flow rate and productivity index of a pseudo steady state system can be expressed as Eq. (1A-2A) (Dake, 1978).

$$
\begin{gathered}
q=\frac{k h}{\ln \left(\frac{r_{\mathrm{e}}}{r_{\mathrm{w}}}\right)-\frac{3}{4}}\left(p_{\text {ave }}-p_{\mathrm{wf}}\right) \\
J=\frac{k h}{\ln \left(\frac{r_{\mathrm{e}}}{r_{\mathrm{w}}}\right)-\frac{3}{4}}
\end{gathered}
$$

where $J$ is the productivity index; $q$ is the flow rate, STB/d.

We use a simple two-layer model filled with oil with constant compressibility. Under pseudo-steady state conditions (i.e. closed system), we can express pressure as a function of time (Dake, 1978) using Eqs. (3A) to (7A):

$$
\begin{aligned}
& c_{\mathrm{t}}=-\frac{1}{v_{\mathrm{p}}} \frac{\partial v_{\mathrm{p}}}{\partial p_{\text {ave }}} \\
& -c_{\mathrm{t}} v_{\mathrm{p}} \frac{\mathrm{d} p_{\text {ave }}}{\mathrm{d} t}=\frac{\mathrm{d} v_{\mathrm{p}}}{\mathrm{d} t} \\
& \frac{\mathrm{d} v_{\mathrm{p}}}{\mathrm{d} t}=q=J\left(p_{\text {ave }}-p_{\mathrm{wf}}\right) \\
& \int_{p_{\text {init }}}^{p} \frac{\mathrm{d} p_{\text {ave }}}{p_{\text {ave }}-p_{\text {wf }}}=\int_{0}^{t}-\frac{J}{c_{\mathrm{t}} v_{\mathrm{p}}} \mathrm{d} t
\end{aligned}
$$

where $c_{\mathrm{t}}$ is the total compressibility; $p_{\text {ave }}$ is the average pressure, $\mathrm{psi} ; p_{\mathrm{wf}}$ is the bottom hole pressure, psi.

By solving Eq. (6A), we can express pressure changes in each layer. i.e., in the second layer as:

$$
p_{2}-p_{\text {wf }}=\left(p_{\text {init }}-p_{\text {wf }}\right) \exp \left(\frac{-J_{2}}{c_{\mathrm{t}} v_{\mathrm{p} 2}} t\right)
$$

where $p_{\text {init }}$ is the initial reservoir pressure, psi.

It is worth mentioning that Eq. (7A) cannot be solved analytically unless we assume that $c_{\mathrm{t}} v_{\mathrm{p}}$ is not a function of time. Other researchers (Fetkovich (1980) have made similar assumption that change of hydrocarbon pore volume with pressure is linear (Eq. (3A)).

For no-crossflow condition between layers and under constant bottom hole pressure, the cumulative production can be written as Eq. (8A):

$$
\begin{aligned}
\int_{0}^{t} q_{i} \mathrm{~d} t & =\int_{0}^{t} J_{i}\left(p_{i}-p_{\mathrm{wf}}\right) \mathrm{d} t=\int_{0}^{t} J_{i} C\left(\exp \left(\frac{-J_{i}}{c_{\mathrm{t}} v_{\mathrm{p} i}} t\right)\right) \mathrm{d} t \\
& =C c_{\mathrm{t}} v_{\mathrm{p} i}\left(1-\exp \left(\frac{-J_{i}}{c_{\mathrm{t}} v_{\mathrm{p} i}} t\right)\right)
\end{aligned}
$$

where $C$ is constant $\left(p_{\text {init }}-p_{\mathrm{wf}}\right) ; p_{i}$ is the average pressure of $i$ th layer, psi; $c_{\mathrm{t}}$ is compressibility; and $v_{\mathrm{p}}$ is the hydrocarbon pore volume, $\mathrm{ft}^{3}$.

For the case of no-crossflow in the system, the flow rate of each layer can be written individually as a function of time. The optimum upscaled (coarse) model should be able to reproduce the production behavior similar to the original twolayer model. This can be written mathematically as Eq. (9A).

$$
\int_{0}^{t}\left(q_{1}(t)+q_{2}(t)-q_{\text {eff }}(t)\right) \mathrm{d} t=0
$$

where $q_{\text {eff }}$ is the production rate from the upscaled model, STB/d. Eq. (9A) can be simplified and rewritten as Eq. (10A):

$$
\begin{aligned}
& C c_{\mathrm{t}} v_{\mathrm{p} 1}\left(1-\exp \left(\frac{-J_{1}}{c_{\mathrm{t}} v_{\mathrm{p} 1}} t\right)\right)+C c_{\mathrm{t}} v_{\mathrm{p} 2}\left(1-\exp \left(\frac{-J_{2}}{c_{\mathrm{t}} v_{\mathrm{p} 2}} t\right)\right) \\
& =C c_{\mathrm{t}}\left(v_{\mathrm{p} 1}+v_{\mathrm{p} 2}\right)\left(1-\exp \left(\frac{-J_{\mathrm{eff}}}{c_{\mathrm{t}}\left(v_{\mathrm{p} 1}+v_{\mathrm{p} 2}\right)} t\right)\right)
\end{aligned}
$$

Eq. (10A) will give the ability to determine how effective productivity index of the coarse model $\left(J_{\text {eff }}\right)$ is changing with time in terms of production profile behavior of fine scale layers:

$$
J_{\text {eff }}=\frac{-c_{\mathrm{t}}\left(v_{\mathrm{p} 1}+v_{\mathrm{p} 2}\right)}{t} \ln \left(\frac{v_{\mathrm{p} 1} \exp \left(\frac{-J_{1}}{c_{\mathrm{t}} v_{\mathrm{p} 1}} t\right)+v_{\mathrm{p} 2} \exp \left(\frac{-J_{2}}{c_{\mathrm{t}} v_{\mathrm{p} 2}} t\right)}{v_{\mathrm{p} 1}+v_{\mathrm{p} 2}}\right)
$$

Alternatively, we can also define the productivity index for the upscaled model using effective permeability as:

$$
J_{\text {eff }}=\frac{k_{\text {eff }}\left(h_{1}+h_{2}\right)}{\ln \left(\frac{r_{\mathrm{e}}}{r_{\mathrm{w}}}\right)-\frac{3}{4}}
$$


So from Eq. (11A) and (12A), $k_{\text {eff }}$ can be obtained analytically as a function of time:

$$
\begin{aligned}
k_{\mathrm{eff}}= & -\frac{\ln \left(\frac{r_{\mathrm{e}}}{r_{\mathrm{w}}}\right)-\frac{3}{4}}{h_{1}+h_{2}} \times \frac{c_{\mathrm{t}}\left(v_{\mathrm{p} 1}+v_{\mathrm{p} 2}\right)}{t} \times \\
& \ln \left(\frac{v_{\mathrm{p} 1} \exp \left(\frac{-J_{1}}{c_{\mathrm{t}} v_{\mathrm{p} 1}} t\right)+v_{\mathrm{p} 2} \exp \left(\frac{-J_{2}}{c_{\mathrm{t}} v_{\mathrm{p} 2}} t\right)}{v_{\mathrm{p} 1}+v_{\mathrm{p} 2}}\right)
\end{aligned}
$$

where $r_{\mathrm{e}}$ is the external radius, $\mathrm{ft}$; $r_{\mathrm{w}}$ is the wellbore radius, $\mathrm{ft}$.

For tracking the behavior of effective permeability of an upscaled model at limiting cases $(t=0$ and $t=\infty)$ Eq. (11A) should be simplified. For late time $(t=\infty)$, Eq. (11A) is simplified into two parts and then the behavior of each part is evaluated separately:

$$
\begin{aligned}
& \lim _{t \rightarrow \infty} \frac{\ln \left(\frac{v_{\mathrm{p} 1} \exp \left(\frac{-J_{1}}{c_{\mathrm{t}} v_{\mathrm{p} 1}} t\right)+v_{\mathrm{p} 2} \exp \left(\frac{-J_{2}}{c_{\mathrm{t}} v_{\mathrm{p} 2}} t\right)}{v_{\mathrm{p} 1}+v_{\mathrm{p} 2}}\right)}{t} \\
& =\lim _{t \rightarrow \infty} \frac{\ln _{\mathrm{p} 1}\left(\exp \left(\frac{-J_{1}}{c_{\mathrm{t}} v_{\mathrm{p} 1}} t\right)+v_{\mathrm{p} 2} \exp \left(\frac{-J_{2}}{c_{\mathrm{t}} v_{\mathrm{p} 2}} t\right)\right)}{t} \\
& -\lim _{t \rightarrow \infty} \frac{\ln \left(v_{\mathrm{p} 1}+v_{\mathrm{p} 2}\right)}{t} \\
& =\lim _{t \rightarrow \infty} \frac{\ln \left(v_{\mathrm{p} 1} \exp \left(\frac{-J_{1}}{c_{\mathrm{t}} v_{\mathrm{p} 1}} t\right)+v_{\mathrm{p} 2} \exp \left(\frac{-J_{2}}{c_{\mathrm{t}} v_{\mathrm{p} 2}} t\right)\right)}{t}-0
\end{aligned}
$$

Unfortunately the result of Eq. (14A) is not obvious for $t=\infty$. For solving it, L'Hopital's rule can be applied to Eq. (14A):

For convenience let us define new variables as:

$$
A=\frac{-J_{1}}{c_{\mathrm{t}} v_{\mathrm{p} 1}}, \quad a=v_{\mathrm{p} 1}, \quad B=\frac{-J_{2}}{c_{\mathrm{t}} v_{\mathrm{p} 2}}, \quad b=v_{\mathrm{p} 2}
$$

Our main purpose is to calculate the limit of Eq. (15A)

$$
\lim _{t \rightarrow \infty} \frac{\ln (a \exp (A t)+b \exp (B t))}{t}
$$
(16A)

Applying L'Hopital's rule to Eq. (15A) will result to Eq.

$$
\lim _{t \rightarrow \infty} \frac{a A \exp (A t)+b B \exp (B t)}{a \exp (A t)+b \exp (B t)}
$$

For long time periods, this equation does not reduce to a limiting value. Let us multiply both numerator and denominator by $\exp (-A t)$. From Eq. (17A) it can be seen that for very late time the answer is simply the largest value between $A$ and $B$.

$$
\lim _{t \rightarrow \infty} \frac{a A \exp ((A-B) t)+b B}{a \exp ((A-B) t)+b}=\left\{\begin{array}{lll}
A & \text { if } & A>B \\
B & \text { if } & A<B
\end{array}\right\}
$$

Even if we multiply both sides by $\exp (-B t)$ the result is exactly the same:

$$
\lim _{t \rightarrow \infty} \frac{a A \exp ((A-B) t)+b B}{a \exp ((A-B) t)+b}=\left\{\begin{array}{lll}
A & \text { if } & A>B \\
B & \text { if } & A<B
\end{array}\right\}
$$

For the case where $t=0$ again we rewrite Eq. (14A) as:

$$
\begin{aligned}
& \lim _{t \rightarrow 0} \frac{\ln \left(\frac{v_{\mathrm{p} 1} \exp \left(\frac{-J_{1}}{c_{\mathrm{t}} v_{\mathrm{p} 1}} t\right)+v_{\mathrm{p} 2} \exp \left(\frac{-J_{2}}{c_{\mathrm{t}} v_{\mathrm{p} 2}} t\right)}{v_{\mathrm{p} 1}+v_{\mathrm{p} 2}}\right)}{t} \\
& =\lim _{t \rightarrow 0} \frac{\ln \left(\frac{a \exp (A t)+b \exp (B t)}{a+b}\right)}{t}
\end{aligned}
$$

After applying L-Hopital's rule and multiplying by $\exp (-B t)$ the final answer will be:

$$
\lim _{t \rightarrow 0} \frac{a A \exp ((A-B) t)+b B}{a \exp ((A-B) t)+b}=\frac{a A+b B}{a+b}
$$

In summary, the limit of above expression is the largest value between $A$ and $B$ as time goes to infinity and weighted arithmetic average of $A$ and $B$ as time goes to zero. Finally by substituting the answer of Eq. (17A) $(A$ or $B)$ into Eq. (13A), the value of effective permeability can be evaluated at late time. As time goes to infinity:

$$
\begin{aligned}
k_{\text {eff }}= & -\frac{\ln \left(\frac{r_{\mathrm{e}}}{r_{\mathrm{w}}}\right)-\frac{3}{4}}{h_{1}+h_{2}} \times \operatorname{ct}\left(v_{\mathrm{p} 1}+v_{\mathrm{p} 2}\right) \times\left(\frac{\mathrm{J}}{c_{\mathrm{t}} v_{\mathrm{p}}}\right)_{\min } \\
k_{\mathrm{eff}}= & \frac{\ln \left(\frac{r_{\mathrm{e}}}{r_{\mathrm{w}}}\right)-\frac{3}{4}}{h_{1}+h_{2}} \times \operatorname{ct}\left(v_{\mathrm{p} 1}+v_{\mathrm{p} 2}\right) \times\left(\frac{k h}{\left(\ln \left(\frac{r_{\mathrm{e}}}{r_{\mathrm{w}}}\right)-\frac{3}{4}\right) c_{\mathrm{t}} v_{\mathrm{p}}}\right)_{\min } \\
= & \left(\frac{k h}{v_{\mathrm{p}}}\right)_{\min } \frac{v_{\mathrm{p} 1}+v_{\mathrm{p} 2}}{h_{1}+h_{2}}
\end{aligned}
$$

where min represents the layer index that has minimum value of productivity index over pore volume (remember that $A$ and $B$ are negative values). When $t$ goes to zero, the answer of Eq. (20A) (effective productivity of the coarse model, using Eq. 
(13A)) could be written as Eq. (23A):

$$
k_{\text {eff }}=-\frac{\ln \left(\frac{r_{\mathrm{e}}}{r_{\mathrm{w}}}\right)-\frac{3}{4}}{h_{1}+h_{2}} \times c t\left(v_{\mathrm{p} 1}+v_{\mathrm{p} 2}\right) \times \frac{a A+b B}{a+b}
$$

Substituting parameters " $a$ " and " $A$ " we will have:

$$
k_{\mathrm{eff}}=-\frac{\ln \left(\frac{r_{\mathrm{e}}}{r_{\mathrm{w}}}\right)-\frac{3}{4}}{h_{1}+h_{2}} \times \operatorname{ct}\left(v_{\mathrm{p} 1}+v_{\mathrm{p} 2}\right) \times \frac{v_{\mathrm{p} 1} \frac{-J_{1}}{c_{\mathrm{t}} v_{\mathrm{p} 1}}+v_{\mathrm{p} 2} \frac{-J_{2}}{c_{\mathrm{t}} v_{\mathrm{p} 2}}}{v_{\mathrm{p} 1}+v_{\mathrm{p} 2}}
$$

Eq. (24A) could be simplified by substituting productivity index definition for both layers (Eq. (2A)), so that the effective permeability at very early time can be calculated as
Eq. (24A):

$$
\begin{aligned}
& k_{\text {eff }}=-\frac{\ln \left(\frac{r_{\mathrm{e}}}{r_{\mathrm{w}}}\right)-\frac{3}{4}}{h_{1}+h_{2}} \times\left(v_{\mathrm{p} 1}+v_{\mathrm{p} 2}\right) \\
& \times\left(\begin{array}{c}
\left.\frac{k h}{\ln \left(\frac{r_{\mathrm{e}}}{r_{\mathrm{w}}}\right)-\frac{3}{4}}\right)_{1}+\left(\frac{k h}{\ln \left(\frac{r_{\mathrm{e}}}{r_{\mathrm{w}}}\right)-\frac{3}{4}}\right)_{2} \\
-\frac{v_{\mathrm{p} 1}+v_{\mathrm{p} 2}}{h_{1}}
\end{array}\right) \\
& =\frac{k_{1} h_{1}+k_{2} h_{2}}{h_{1}+h_{2}}
\end{aligned}
$$

which is simply the arithmetic weighted average.

(Edited by Sun Yanhua) 